\title{
Self-harm in young people
}

\section{Ellen Townsend}

Correspondence to Dr Ellen Townsend, Self-Harm Research Group, School of Psychology, University of Nottingham, University Park, Nottingham NG7 2RD, UK; Ellen.Townsend@nottingham.ac.uk

\begin{abstract}
Self-harm is a common problem among young people with many presenting to clinical services via general hospitals, but many more do not come to the attention of clinical services at all. Self-harm is strongly associated with completed suicide so it is extremely important that patients are assessed and treated for this problem effectively. Despite the scale of the problem in young people, there is a very limited evidence base on what interventions may help them to recover from self-harm. The evidence is discussed here and some recommendations are made about how to engage clinically with young people who self-harm from assessment to therapeutic intervention.
\end{abstract}

\section{WHAT IS SELF-HARM AND WHAT IS THE SCALE OF THE PROBLEM IN YOUNG PEOPLE?}

Self-harm (self-poisoning or self-injury regardless of the motivation or intent associated with the act) is common in young people. ${ }^{1}$ Here, 'young people' refers to anyone from age 11 up to the age of 25 years, since psychologists and neuroscientists now suggest this as the most appropriate cut-off for late-adolescence. Hospital-based data demonstrate that self-harm is most common in 15-24-year-olds, with females presenting more frequently than males. ${ }^{2}$ However, hospital-based statistics are likely to be the 'tip of the iceberg', with many more episodes occurring in the community that do not come to medical attention; community-based studies estimate that around $10 \%$ of young people have self-harmed. ${ }^{3}$

Self-harm is often repeated; in young people presenting to hospital for self-harm around half will have a history of prior harm and $18 \%$ will repeat the behaviour within a year (and present to hospital) again. ${ }^{1}$ Self-harm is strongly linked to completed suicide with $40-60 \%$ of those who die by suicide having self-harmed in the past, making it the strongest predictor of eventual suicide. ${ }^{4}$ In one consecutive case series study, $80 \%$ of young people who died by suicide had self-harmed in the preceding year. $^{5}$

As Owens et al ${ }^{4}$ put it, 'suicide risk among self-harm patients is hundreds of times higher than the general population'. Suicide is the second commonest cause of death in young people globally. ${ }^{6}$ Importantly, and of significant clinical relevance, is the recent research demonstrating that self-cutting in young people who self-harm is a significant risk factor for completed suicide as is being male, having previously self-harmed and having received psychiatric treatment. ${ }^{7}$ Given these associations, it is vitally important that young people seen by a clinician (in any setting) for self-harm are taken seriously and are cared for competently and compassionately. ${ }^{8}$

In this review, I discuss studies that were identified via Web of Knowledge, MEDLINE and Psyclnfo using combinations of the search terms: self-harm* ${ }^{*}$ self harm* ${ }^{*}$, randomized controlled trial ${ }^{*}$, randomised controlled trial ${ }^{*}$, attempted suicide* and intervent* (update: May 2014).

\section{WHY DO YOUNG PEOPLE SELF-HARM?}

Research on understanding the 'why's' of self-harmful behaviour is still quite limited, ${ }^{9}$ with much research focusing on risk factors for the behaviour rather than the biopsychosocial mechanisms driving it. Contemporary theories emphasise psychological mechanisms such as the coping and emotion regulation functions associated with selfharm. ${ }^{10}$ Other theories acknowledge the complex interplay of psychological, biological and social factors involved and the 'escape' function of the behaviour. ${ }^{11}$ Indeed, self-reported motives from young people reveal that wanting to escape or get relief from unbearable feelings or an unbearable state of mind/situation are commonly reported motives for self-harm with some young people wishing to die as a result of their act. ${ }^{3}{ }^{12} \mathrm{~A}$ greater understanding of the psychological mechanisms associated with self-harm, such as self-esteem, impulsivity, hopelessness, depression and entrapment are important when determining vulnerability to self-harm across all ages, ${ }^{3} 1213$ with currently influential theories emphasising the importance of the stress-diathesis between key risk factors. ${ }^{11}$

Indeed, young people who self-harm experience negative life events and problems, especially with relationships with partners, families and friends. ${ }^{1}$ Moreover, knowing someone else (friend or family member) who has self-harmed increases the risk of engaging in self-harm significantly. ${ }^{13}$ One limitation of many studies in the literature is that they are cross-sectional in nature, which limits their predictive utility in determining who may be at risk from initiating or repeating self-harm or completing suicide.

\section{HOW SHOULD YOUNG PEOPLE WHO SELF-HARM BE ASSESSED?}

National Institute for Health and Care Excellence (NICE) guidance on the longer term management of self-harm indicates that all individuals who self-harm should be offered a comprehensive assessment both for risk of further episodes and clinical, social, psychosocial and physical needs. ${ }^{14}$ Assessment of depression, hopelessness and current/past self-harm and suicidal intent are particularly important given their association with further self-harm and suicide described earlier. In some high-risk groups (such as young offenders), other psychological factors such as anxiety and self-esteem are also important aspects to consider. ${ }^{9}$ Excellent guidance on assessing suicidal risk and intent associated with self-harm can be found in the refreshing 'suicide mitigation' approach developed by Cole-King et al..$^{8}$ During assessment of subsequent risk of fatal and non-fatal repetition, the method of self-harm used is important to consider since robust longitudinal data indicate that cutting is a significant risk factor for completed suicide in children and adolescents. ${ }^{7}$ Finally, the use of risk assessment tools alone to predict the risk of future self-harm or suicide, or to determine who should receive further treatment is not recommended owing to the low predictive utility of these scales. ${ }^{14}$ They may, however, be used in tandem with, or to guide, a structured psychosocial history and risk assessment as set-out in the NICE guidance. ${ }^{14}$

Qualitative data reveal that young people who self-harm find it very difficult to talk about their self-harm and suicidal feelings ${ }^{15}$ and do not feel 'listened to' when they do. ${ }^{16}$ Being listened to is viewed as important, especially in females. ${ }^{17}$ Indeed, experiencing negativity and judgmental attitudes from clinicians can be very detrimental therapeutically, ${ }^{18}$ and (unfortunately) robust systematic review evidence suggests that attitudes towards self-harm among clinical staff are negative. ${ }^{19}$ Training on self-harm awareness and suicide is vital, but lacking for many frontline staff $^{20-22}$ who feel that they do not possess the ability to deal with self-harm effectively. ${ }^{22-24}$ This is especially worrying given the fact that 
a recent systematic review shows that 1 in 25 patients presenting to hospital emergency departments for self-harm will die by suicide in the following 5 years. ${ }^{25}$ Crucially, educating doctors is one of the few suicide prevention strategies shown to reduce suicide rates significantly. ${ }^{26}$ Happily, even very short-term training can alter and improve staff attitudes significantly. ${ }^{27}$

A randomised controlled trial (RCT) has demonstrated that 'therapeutic assessment' has a significant and sustained impact on engagement with treatment in young people who self-harm. ${ }^{28}$ Here a standard assessment (psychosocial history and risk assessment) based on NICE guidance was compared with a therapeutic assessment which included the standard assessment plus a brief (30 min) intervention involving a number of elements including the joint drawing of a diagram of 'reciprocal roles, core pain and maladaptive procedures', identification of a target problem, exploring motivation for change and 'exits' out of the behaviour. An 'understanding letter' was then sent describing the diagram and the exits discussed in the assessment. Those receiving the therapeutic assessment showed a significant increase in treatment engagement at 2-year follow-up compared with those receiving a standard assessment. The significant effect on therapeutic engagement may have occurred because of an increase in clients' 'hopes and expectations from self-harm assessment through an unusual reminder' (sending the understanding letter). Alternatively, as the authors note, the increased time spent on therapeutic assessment (around $40 \mathrm{~min}$ ) and/or the enthusiasm of the clinicians may have led to increased engagement. ${ }^{28}$ Another RCT which aimed to increase treatment adherence used a compliance enhancement treatment ${ }^{29}$ and showed that increased adherence was observed in the treated group (eight sessions attended on average) compared with a group randomised to receive standard care (five sessions attended) once 'barriers to services' were controlled for

The importance of receiving a psychosocial assessment (at any age) is highlighted by findings from the Multicentre Study of Self-Harm in England. ${ }^{30}$ Analysis of 10 years of data from 35958 individuals (of all ages) showed that having a psychosocial assessment on presentation to hospital for self-harm was associated with a $40 \%$ lower risk of repetition. Thus, it is essential that all young people who present for selfharm receive a detailed psychosocial assessment-it should not be viewed or presented as optional-especially given the high risk of suicide in the 5 years following presentation to hospital. ${ }^{25}$

\section{WHICH INTERVENTIONS ARE RECOMMENDED FOR YOUNG PEOPLE WHO SELF-HARM?}

The evidence base for effective interventions for adolescents who selfharm is extremely limited and few have been evaluated in robust $\mathrm{RCTS}^{31}$ - the gold standard evidence for determining the effectiveness of interventions. A few large trials are also currently underway at the time of writing. The effectiveness of group-based psychotherapy versus treatment as usual (TAU) has been investigated in three RCTs with adolescents aged 12-17 years old with equivocal results. In the original study of developmental group therapy, those receiving group therapy were less likely to have repeated self-harm at follow-up. ${ }^{32}$ In contrast, a study conducted in Australia which tried to replicate this trial failed to do so with more adolescents in the group therapy arm repeating selfharm than in the TAU arm. ${ }^{33}$ The lack of replication could be due to a number of reasons such as less experienced therapists in the later (Australian) trial, differences in the population recruited to the study and the fact that only $57 \%$ of the target sample required for statistical power was reached in the trial. A third large $(n=366)$ trial-the Assessment of Treatment in Suicidal Teenagers (ASSIST; delivered in addition to routine care)-examined a different outcome measure to the previous studies (frequency of repeated self-harm rather than the proportion of each group repeating self-harm). ${ }^{34}$ The data revealed no benefit of adding group therapy to routine care in terms of reducing self-harm frequency. It is unclear why these two later trials failed to replicate the original pilot trial of group therapy. One suggestion is that the findings reflect the improvement of service provision in routine care since the original trial. ${ }^{35}$ Alternatively, this mode of therapy may not adequately deal with the psychological processes that underpin selfharmful behaviour in young people.

Few studies have evaluated the impact of individual psychological therapy with adolescents who self-harm (although there is a growing evidence base for the effectiveness of these for adults). ${ }^{14} 31$ One small pilot study evaluated the impact of a cognitive behavioural treatment against a non-directive supportive treatment. ${ }^{35}$ No differences between the groups were observed in terms of the rate of self-harm at follow-up; however, the trial was very small and likely to be insufficiently powered to detect such differences. By contrast, a significant impact on self-harmful behaviour was observed in an RCT that compared mentalisation-based therapy (a 12-month-long psychodynamic psychotherapy programme rooted in attachment theory) with TAU. Adolescents who received mentalisation-based therapy were significantly less likely to have self-harmed at follow-up than those assigned to $\mathrm{TAU}^{36}$

Home-based family therapy has also been tried for young people who self-harm but did not reduce self-harm at follow-up; ${ }^{37}$ a larger trial of family therapy is currently underway. ${ }^{38}$ It is notable that no RCTs or cohort studies have evaluated harm reduction or minimisation for any age group. ${ }^{14}$ Such strategies include harming oneself 'safely', for example, by being aware of the location of veins and arteries when cutting, using a sterile, sharp blade and suggesting alternative behaviours to distract from the urge to self-harm such as pinching, squeezing ice or snapping rubber bands on one's wrist. The evidence for these strategies reviewed in the 2011 NICE guidance ${ }^{14}$ was inconclusive, though some tentative recommendations about harm reduction were made (developing strategies other than self-harm, using less harmful methods and advising that there is no safe way to self-poison). Clinically, however, this approach risks failing to address the motivation and meaning associated with self-harm for each client. Focusing on alternative or safer behaviours in clinical practice, rather than (even briefly) acknowledging and being compassionate about the intense psychological distress that accompanies self-harm, could send a negative, even dismissive, message to young people accessing services for this issue.

\section{RECOMMENDATIONS FOR CLINICAL PRACTICE AND SERVICE PROVISION}

Dealing with clients compassionately in a competent manner through active, non-judgmental listening should be the bedrock of all clinical practice for self-harm, ${ }^{8}$ especially with young people. Given the negative attitudes that persist in clinical practice it is crucial that all frontline staff receive self-harm and suicide awareness training - even briefly. Although the literature lacks clarity about 'what works' in the management of self-harm in adolescents, ${ }^{14}{ }^{31}$ RCT evidence suggests that enhanced assessment (adding briefly delivered extras in addition to routine assessments) may help keep adolescents engaged with treatment, $^{28} 29$ and receiving a psychosocial assessment after self-harm protects against later repetition. ${ }^{30}$ The evidence pertaining to interventions is lacking and it is important to note that no interventions evaluated in RCTs have been replicated independently. ${ }^{39}$ Nonetheless, mentalisation-based therapy is a promising new treatment that appears to significantly reduce self-harm in adolescents ${ }^{36}$ and larger trials of treatments are underway. ${ }^{38}$ NICE guidance for the longer term management of self-harm recommends that brief psychological therapies should be offered, but this recommendation is not based on evidence specific to young people. Thus, theoretically driven, adequately powered 
RCTs of psychological treatments are now urgently required in this field to inform recommendations for clinical practice with young people who self-harm.

Competing interests None.

doi:10.1136/eb-2014-101840

\section{REFERENCES}

1. Hawton K, Bergen H, Casey D, et al. Self-harm in England: a tale of three cities. Multicentre study of self-harm. Soc Psychiatry Psychiatr Epidemiol 2007;42:513-21.

2. Hawton $\mathbf{K}$, Bergen $\mathrm{H}$, Waters $\mathrm{K}$, et al. Epidemiology and nature of self-harm in children and adolescents: findings from the multicentre study of self-harm in England. Eur Child Adolesc Psychiatry 2012;21:369-77.

3. Hawton K, Saunders KEA, Connor RCO. Series suicide 1 self-harm and suicide in adolescents. Lancet 2012;379:2373-82

4. Owens D, Horrocks J, House A. Fatal and non-fatal repetition of self-harm systematic review. Br J Psychiatry 2002;181:193-9.

5. Hawton K, Houston K, Shepperd R. Suicide in young people. Study of 174 cases, aged under 25 years, based on coroners' and medical records. Br J Psychiatry 1999;175:271-6

6. Patton GC, Coffey C, Sawyer SM, et al. Global patterns of mortality in young people: a systematic analysis of population health data. Lancet 2009;374:881-92.

7. Hawton $\mathbf{K}$, Bergen $\mathrm{H}$, Kapur $\mathrm{N}$, et al. Repetition of self-harm and suicide following self-harm in children and adolescents: findings from the multicentre study of self-harm in England. J Child Psychol Psychiatry 2012;53:1212-19.

8. Cole-King A, Green G, Gask L, et al. Suicide mitigation: a compassionate approach to suicide prevention. Adv Psychiatr Treat 2013;19:276-83.

9. Knowles S, Townsend E, Anderson M. Factors associated with self-harm in community-based young offenders: the importance of psychological variables. J Forens Psychiatry Psychol 2011;22:479-95.

10. Chapman AL, Gratz KL, Brown MZ. Solving the puzzle of deliberate self-harm: the experiential avoidance model. Behav Res Ther 2006;44:371-94.

11. O'Connor RC. The integrated motivational-volitional model of suicidal behavior. Crisis 2011;32:295-8

12. O'Connor RC, Rasmussen S, Hawton K. Predicting deliberate self-harm in adolescents: a six month prospective study. Suicide Life Threat Behav 2009:39:364-75.

13. O'Connor RC, Rasmussen S, Hawton K. Distinguishing adolescents who think about self-harm from those who engage in self-harm. $\mathrm{Br} J$ Psychiatry 2012;200:330-5

14. National Collaborating Centre for Mental Health commissioned by the National Institute for Health and Clinical Excellence. Self-harm: Ionger-term management. National Clinical Guideline Number 133. London: The British Psychological Society and the Royal College of Psychiatrists, 2011.

15. Bostik KE, Everall RD. In my mind I was alone: suicidal adolescents' perceptions of attachment relationships. Int J Adv Couns 2006;28:269-87.

16. Mental Health Foundation. Truth hurts - report of the National Inquiry into Self-harm among Young People. Mental Health Foundation, 2006.

17. Fortune S, Sinclair J, Hawton K. Adolescents' views on preventing self-harm. A large community study. Soc Psychiatry Psychiatr Epidemiol 2008;43:96-104.

18. Thompson AR, Powis J, Carradice A. Community psychiatric nurses' experience of working with people who engage in deliberate self-harm. Int J Ment Health Nurs 2008; 17:153-61.
19. Saunders KE, Hawton K, Fortune S, et al. Attitudes and knowledge of clinical staff regarding people who self-harm: a systematic review. J Affect Disord 2012;139:205-16.

20. Crawford T, Geraghty W, Street K, et al. Staff knowledge and attitudes towards deliberate self-harm in adolescents. J Adolesc 2003;26:619-29.

21. McCann T, Clark E, McConnachie S, et al. Accident and emergency nurses' attitudes towards patients who self-harm. Accid Emerg Nurs 2006;14:4-10.

22. Friedman $\mathbf{T}$, Newton $\mathrm{C}$, Coggan $\mathrm{C}$, et al. Predictors of A\&E staff attitudes to self-harm patients who use self-laceration: influence of previous training and experience. J Psychosom Res 2006;60:273-7.

23. Knowles SE, Townsend E, Anderson MP. Youth Justice staff attitudes towards screening for self-harm. Health Soc Care Community 2012;20:506-15.

24. Knowles SE, Townsend E, Anderson MP. "In two minds"-socially motivated self-harm is perceived as less serious than internally motivated: a qualitative study of youth justice staff. J Health Psychol 2013;18:1187-98.

25. Carroll R, Metcalfe C, Gunnell D. Hospital presenting self-harm and risk of fatal and non-fatal repetition: systematic review and meta-analysis. PLOS ONE 2014:e89944.

26. Mann JJ, Haas A, Mehlum L, et al. Suicide prevention strategies. JAMA 2005;294:2064-74

27. Botega NJ, Silva SV, Reginato DG, et al. Maintained attitudinal changes in nursing personnel after a brief training on suicide prevention. Suicide Life Threat Behav 2007:37:145-53.

28. Ougrin D, Boege I, Stahl D, et al. Randomised controlled trial of therapeutic assessment versus usual assessment in adolescents with self-harm: 2-year follow-up. Arch Dis Child 2013;98:772-6.

29. Spirito A, Boergers J, Donaldson D, et al. An intervention trial to improve adherence to community treatment by adolescents after a suicide attempt. J Am Acad Child Adolesc Psychiatry 2002;41:435-42.

30. Kapur N, Steeg S, Webb R, et al. Does clinical management improve outcomes following self-harm? Results from the multicentre study of self-harm in England. PLOS ONE 2013;8:e70434.

31. Hawton K, Townsend E, Arensman E, et al. Psychosocial and pharmacological treatments for deliberate self harm (review). Cochrane Libr 2009;(4).

32. Wood A, Trainor G, Rothwell J, et al. Randomized trial of group therapy for repeated deliberate self-harm in adolescents. J Am Acad Child Adolesc Psychiatry 2001:40:1246-53.

33. Hazell PL, Martin G, McGill K, et al. Group therapy for repeated deliberate self-harm in adolescents: failure of replication of a randomized trial. J Am Acad Child Adolesc Psychiatry 2009;48:662-70.

34. Green JM, Wood AJ, Kerfoot MJ, et al. Group therapy for adolescents with repeated self harm: randomised controlled trial with economic evaluation. BMJ 2011;342:d682

35. Donaldson D, Spirito A, Esposito-Smythers C. Treatment for adolescents following a suicide attempt: results of a pilot trial. J Am Acad Child Adolesc Psychiatry 2005:44:113-20.

36. Rossouw TI, Fonagy P. Mentalization-based treatment for self-harm in adolescents: a randomized controlled trial. J Am Acad Child Adolesc Psychiatry 2012;51:1304-13

37. Harrington R, Kerfoot M, Dyer $\mathrm{E}$, et al. Randomized trial of a home-based family intervention for children who have deliberately poisoned themselves. J Am Acad Child Adolesc Psychiatry 1998;37:512-18.

38. Cottrell DJ. ISRCTN Register. 2014. http://www.controlled-trials.com/ ISRCTN59793150

39. Ougrin D, Tranah T, Leigh E, et al. Practitioner review: self-harm in adolescents $J$ Child Psychol Psychiatry 2012;53:337-50 